\title{
Adolescents' interest in trying flavoured e-cigarettes
}

\author{
J K Pepper, ${ }^{1,2}$ K M Ribisl, ${ }^{1,3}$ N T Brewer ${ }^{1,3}$
}

${ }^{1}$ Department of Health Behavior, Gillings School of Global Public Health, University of North Carolina, Chapel Hill, North Carolina, USA ${ }^{2}$ RTI International, Research Triangle Park, North Carolina, USA

${ }^{3}$ Lineberger Comprehensive Cancer Center, University of North Carolina, Chapel Hill, North Carolina, USA

\section{Correspondence to} Dr JK Pepper, RTI International, 3040 East Cornwallis Rd., Research Triangle Park, NC 27709, USA; jpepper@rti.org

Received 17 May 2016 Revised 30 June 2016 Accepted 7 July 2016 Published Online First 15 September 2016

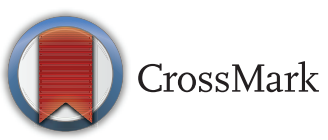

To cite: Pepper JK, Ribisl KM, Brewer NT. Tob Control 2016:25:ii62-ii66.

\begin{abstract}
Background More US adolescents use e-cigarettes than smoke cigarettes. Research suggests flavoured e-cigarettes appeal to youth, but little is known about perceptions of and reasons for attraction to specific flavours.
\end{abstract}

Methods A national sample of adolescents $(n=1125)$ ages 13-17 participated in a phone survey from November 2014 to June 2015. We randomly assigned adolescents to respond to survey items about 1 of 5 e-cigarette flavours (tobacco, alcohol, menthol, candy or fruit) and used regression analysis to examine the impact of flavour on interest in trying e-cigarettes and harm beliefs.

Results Adolescents were more likely to report interest in trying an e-cigarette offered by a friend if it were flavoured like menthol $(\mathrm{OR}=4.00,95 \% \mathrm{Cl} 1.46$ to 10.97), candy ( $\mathrm{OR}=4.53,95 \% \mathrm{Cl} 1.67$ to 12.31$)$ or fruit (OR=6.49, 95\% Cl 2.48 to 17.01) compared with tobacco. Adolescents believed that fruit-flavoured e-cigarettes were less harmful to health than tobaccoflavoured e-cigarettes $(p<0.05)$. Perceived harm mediated the relationship between some flavours and interest in trying e-cigarettes. A minority of adolescents believed that e-cigarettes did not have nicotine (14.6\%) or did not know whether they had nicotine (3.6\%); these beliefs did not vary by flavour.

Discussion Candy-flavoured, fruit-flavoured and menthol-flavoured e-cigarettes appeal to adolescents more than tobacco-flavoured or alcohol-flavoured e-cigarettes. This appeal is only partially explained by beliefs about reduced harm. Given adolescents' interest in trying e-cigarettes with certain flavours, policymakers should consider restricting advertisements promoting flavoured products in media that reach large numbers of young people. Future research should examine other reasons for the appeal of individual flavours, such as novelty and perceived luxury.

\section{INTRODUCTION}

According to industry documents, cigarette manufacturers have long known that flavoured products appeal to youth and have used flavours to target young people. ${ }^{1}$ Flavours influence smoking initiation, increase smoking progression by masking the harsh taste of tobacco products ${ }^{1}$ and are particularly appealing to younger users. ${ }^{2-4}$ Most adolescents who have experimented with tobacco products began with flavoured products. ${ }^{4}$ The Food and Drug Administration (FDA) Center for Tobacco Products banned the sale of flavoured cigarettes (other than tobacco and menthol flavours) in the USA in 2009.5 In an earlier draft of proposed regulations in 2014, FDA intended to extend the flavour ban to electronic cigarettes (e-cigarettes), but the 2016 final version of the regulation struck this provision. ${ }^{6}$
E-cigarettes are surging in popularity among youth (including children and teenagers), ${ }^{7}$ perhaps partly due to the availability and variety of flavours. E-cigarette liquids come in thousands of flavours. ${ }^{8}$ Although flavoured e-cigarettes are popular with all age groups, they appear to be particularly enticing to adolescents. In the most recently released data from the Population Assessment of Tobacco and Health (PATH) study, $85 \%$ of current e-cigarette users ages 12-17 use flavoured versions of ecigarettes compared with only $63 \%$ of adults ages 25 and older. ${ }^{9}$ Data from the National Youth Tobacco Survey (NYTS) show that among youth who used any tobacco product in 2014, 63\% (1.58 million) had used a flavoured e-cigarette. ${ }^{10}$ Moreover, two recent surveys with large national samples found that the vast majority $(79-81 \%)$ of youth ever-users of e-cigarettes initiated e-cigarette use with a flavoured product. ${ }^{49}$ The availability of appealing flavours is often the first ${ }^{9}$ or second ${ }^{11}$ most popular reason for using e-cigarettes among youth and young adults. Flavoured e-cigarettes are a public health concern not only because they may contribute to youth experimentation, but also because the chemicals that serve as flavourants may pose their own health hazards. ${ }^{12} 13$

With the exception of a handful of studies that used potentially problematic control groups (eg, comparing adolescents who do not smoke tobacco cigarettes to tobacco cigarette smoking adults) ${ }^{14}$ or conditions (eg, comparing flavoured ecigarettes to an unspecified type of e-cigarette), ${ }^{15}$ the emerging literature generally links flavoured e-cigarettes and youth appeal. ${ }^{911}$ Our study built on prior work by examining interest in specific sweet and menthol flavours, not a generic category of flavoured e-cigarettes, and by using an experimental design among a national sample of adolescents. We also extended the literature by exploring possible explanations for interest. Specifically, we explored whether perceived harm mediated the relationship between flavours and interest in use and observed whether this relationship varied by specific flavour.

\section{METHODS}

\section{Participants}

From November 2014 to June 2015, the Carolina Survey Research Laboratory (CSRL) at the University of North Carolina recruited a probability sample of 1125 US adolescents for a telephone survey using random-digit-dial landline and cell phone frames. CSRL oversampled counties with higher prevalence of low-income respondents and cigarette smokers. To be eligible for the study, adolescents had to be ages 13-17 and speak English or Spanish. Interviewers first obtained verbal consent from adolescents' parents or guardians and then verbal assent from the adolescents. The response 
rate among adolescents was 66\% (American Association for Public Opinion Research formula 4). The Institutional Review Board at the University of North Carolina approved the study.

\section{Procedures and measures}

Adolescents first heard the following description of e-cigarettes: 'The next few questions are about electronic or e-cigarettes and other vaping devices, such as e-hookah and vape pens. Popular brands include Blu, Vuse, NJOY and Flavour Vapes'. The computer-assisted telephone interviewing software then randomly assigned adolescents to one of five flavour conditions: 'tobacco' (the control condition); 'alcohol, like scotch or champagne'; 'menthol'; 'candy, like chocolate or vanilla'; or 'fruit, like cherry or peach'. Adolescents responded about their interest in trying an e-cigarette in that flavour: 'If one of your best friends were to offer you an e-cigarette or other vaping device with (flavour condition), would you use it?' We recoded their responses on this primary outcome variable so that a value of 0 corresponded to 'definitely no' or 'probably no' and a value of 1 corresponded to 'definitely yes' or 'probably yes'. We assessed their perceptions of health risks with the item 'If you regularly used an e-cigarette or other vaping device with (flavour condition), how harmful to your health do you think it would be?' Response options were 'not at all' (coded as 1), 'moderately' (2), 'very' (3) or 'extremely' (4). In addition, we assessed adolescents' perceptions of whether the product in their assigned flavour condition had any nicotine ('no' coded as 0 and 'yes' as 1 ).

Demographic measures included sex, race/ethnicity, age, region and mothers' education (categories: high school or less; some college or Associate's degree; Bachelor's degree or more; and don't know). We recoded to create three categories of ecigarette users: never-users, ever-users (used $\geq 1$ time but not in the past 30 days) and current users (used $\geq 1$ time in the past 30 days). We did not exclude ever or current e-cigarette users as these adolescents could still have differing levels of interest in using particular flavours and beliefs about the harm of particular flavours. We similarly recoded cigarette smoking status to categorise respondents as never-smokers, ever-smokers (smoked $\geq 1$ time but not in the past 30 days) and current smokers (smoked $\geq 1$ time in the past 30 days).

\section{Data analysis}

To check whether random assignment created demographically equivalent groups by flavour condition, we used $\chi^{2}$ tests for categorical demographic variables (sex, race/ethnicity, region, mothers' education, e-cigarette use and cigarette smoking) and linear regression for the continuous demographic variable (age). Using logistic regression, we examined the effects of flavour condition on interest in trying e-cigarettes if offered by a friend and on beliefs about whether the e-cigarette contained nicotine. We used linear regression to assess the association between flavour and perceived harm. Tobacco flavour was the reference category in these three regressions. Next we assessed whether perceived harm mediated the relationship between flavour and interest in trying e-cigarettes and used a Sobel test to examine the significance of the mediation effect. Finally, we conducted multivariable logistic regression of willingness to try e-cigarettes including flavour, perceived harm, cigarette smoking, e-cigarette use and other demographic variables as predictors. In both the mediation analysis and multivariable logistic regression, we dichotomised 'flavour' as menthol/candy/fruit versus tobacco/ alcohol because of our empirical findings from the bivariate analysis on which flavours interested adolescents more than tobacco flavour. We conducted analyses using Stata V.12. Regression coefficients are presented as ORs or standardised $\beta$. Analyses used two-tailed statistical tests and a critical $\alpha$ of 0.05 .

\section{RESULTS \\ Participants}

Adolescents' mean age was 15.1 years, and half were female (table 1). The majority of participants were non-Hispanic white (76\%) and reported that their mothers had attended at least some college $(65 \%)$. Most adolescents had never smoked cigarettes $(89 \%)$ or used e-cigarettes $(85 \%)$, but $4 \%$ were current smokers and $5 \%$ were current e-cigarette users.

\section{Effects of flavour descriptor}

Adolescents reported that, if offered by a friend, they were more likely to try menthol-flavoured $(8.3 \%, \mathrm{OR}=4.00,95 \% \mathrm{CI}$ 1.46 to 10.97$)$, candy-flavoured $(9.3 \%, \mathrm{OR}=4.53,95 \%$ CI 1.67 to 12.31$)$ or fruit-flavoured e-cigarettes $(12.8 \%, \mathrm{OR}=6.49$, $95 \%$ CI 2.48 to 17.01$)$ compared with tobacco-flavoured ecigarettes (2.2\%; figure 1$)$. Interest in trying alcohol flavours (4.0\%) and tobacco flavour did not differ. Adolescents perceived fruit-flavoured e-cigarettes to be less harmful than tobaccoflavoured ones (mean 2.71 vs $2.87, \beta=-0.08$, p $<0.05$ ), but they did not view the other flavours as more harmful (alcohol=3.00, menthol=2.87 and candy=2.78). Flavour was not associated with perceived presence of nicotine. A minority of participants reported that e-cigarettes, regardless of flavour, had no nicotine $(14.6 \%)$, or they were not sure if e-cigarettes had nicotine (3.6\%).

\section{Table 1 Participant characteristics $(n=1125)$}

\begin{tabular}{|c|c|c|}
\hline Characteristic & $\mathbf{n}$ & Per cent \\
\hline \multicolumn{3}{|l|}{ Sex } \\
\hline Male & 561 & 49.9 \\
\hline Female & 564 & 50.1 \\
\hline Age: mean (SD) & $15.1(1.4)$ & \\
\hline \multicolumn{3}{|l|}{ Race/ethnicity } \\
\hline Non-Hispanic white & 859 & 76.4 \\
\hline Non-Hispanic other race & 182 & 16.2 \\
\hline Hispanic & 84 & 7.5 \\
\hline \multicolumn{3}{|l|}{ Mother's education } \\
\hline High school or less & 218 & 19.4 \\
\hline Some college or Associate's degree & 186 & 16.5 \\
\hline Bachelor's degree or more & 545 & 48.4 \\
\hline Don't know & 176 & 15.6 \\
\hline \multicolumn{3}{|l|}{ Region } \\
\hline Northeast & 154 & 13.7 \\
\hline Midwest & 285 & 25.3 \\
\hline South & 545 & 48.4 \\
\hline West & 141 & 12.5 \\
\hline \multicolumn{3}{|l|}{ Cigarette smoking } \\
\hline Never-smoker & 1004 & 89.2 \\
\hline Ever-smoker* & 80 & 7.1 \\
\hline Current smokert & 41 & 3.6 \\
\hline \multicolumn{3}{|l|}{ E-cigarette use } \\
\hline Never-user & 958 & 85.2 \\
\hline Ever-user* & 109 & 9.7 \\
\hline Current usert & 58 & 5.2 \\
\hline
\end{tabular}




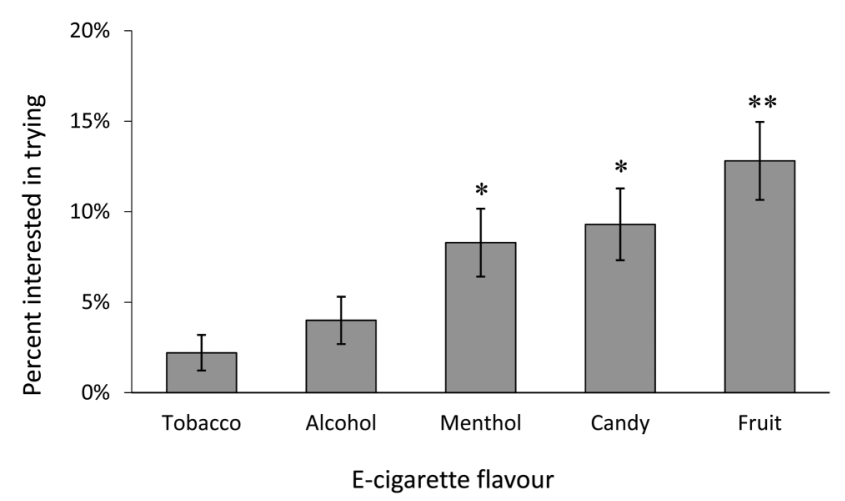

Figure 1 Interest in trying an e-cigarette if offered by a friend. Error bars show SEs. ${ }^{*} p<0.01$ and ${ }^{* *} p<0.001$ different from tobacco flavour.

\section{Mediation}

Perceptions of e-cigarette harm partly explained (ie, mediated) the relationship between flavour and interest in trying ecigarettes if offered by a friend (figure 2). Adolescents believed that menthol-flavoured, candy-flavoured or fruit-flavoured ecigarettes were less harmful than tobacco-flavoured or alcoholflavoured ones $(\beta=-0.15, \mathrm{p}<0.01)$. Greater perceived harmfulness was associated with less interest in trying e-cigarettes $(\mathrm{OR}=0.31,95 \% \mathrm{CI} 0.22$ to 0.43$)$. Controlling for perceived harm reduced the association between flavour and interest in trying (Sobel $\mathrm{z}=2.84, \mathrm{p}<0.01$ ), and the association remained statistically significant $(\mathrm{OR}=3.24,95 \% \mathrm{CI} 1.78$ to 5.90$)$, a pattern of findings that indicates partial mediation.

\section{Multivariable predictors of interest in trying e-cigarettes}

In a multivariable regression, interest in trying e-cigarettes if offered by a friend was correlated with flavour condition (menthol, candy, or fruit vs tobacco or alcohol) and perceived harm as reported above, as well as region, cigarette smoking and e-cigarette use (table 2). Compared with adolescents living in the Midwest (5\% interested), adolescents living in the Northeast were more interested in trying e-cigarettes $(8 \%$; $\mathrm{OR}=3.33$, 95\% CI 1.20 to 9.22 ). Only $3 \%$ of never cigarette smokers were interested in trying e-cigarettes, compared with $30 \%$ of ever-smokers $(\mathrm{OR}=3.16,95 \% \mathrm{CI} 1.45$ to 6.89$)$ and $63 \%$ of current smokers (OR=7.82, 95\% CI 2.86 to 21.32). The pattern was similar for past e-cigarette use. Only $2 \%$ of never e-cigarette users were interested in trying e-cigarettes in the situation described (a particular flavour offered by a friend), whereas $31 \%$ of ever-users $(\mathrm{OR}=12.47,95 \%$ CI 5.89 to 26.41$)$ and $57 \%$ of current users $(\mathrm{OR}=25.75,95 \%$ CI 10.30 to 64.36$)$ would try the product in that scenario. Sex, age, race/ethnicity and mothers' education were not associated with interest in trying e-cigarettes if offered by a friend.

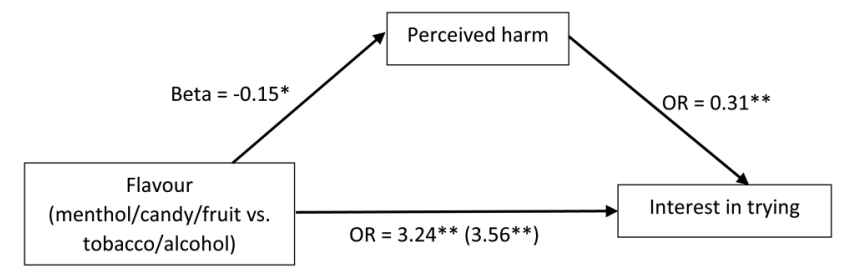

Figure 2 Perceived harm mediates effect of flavour descriptors on interest in trying e-cigarettes. Numbers in parentheses show the association between flavour and interest in trying before controlling for perceived harm. Path values are standardised $\beta$ or ORs. * $p<0.01$, ${ }^{* *} p<0.001$.

\section{DISCUSSION}

Among a national sample of US adolescents, we found that adolescents were more interested in trying e-cigarettes described as having menthol, candy or fruit flavours than tobacco or alcohol flavours. Belief that these sweet and menthol flavours of ecigarettes were less harmful explained some of the difference in interest. Most adolescents believed that e-cigarettes, regardless of flavour, contain nicotine. However, around one in five adolescents did not believe or were unsure of whether e-cigarettes have nicotine, a potential cause for concern.

Interest in trying e-cigarettes was highest for fruit flavours, almost six times higher than interest in tobacco-flavoured ecigarettes. Although menthol and candy flavours were also more appealing than tobacco flavour, alcohol and tobacco did not differ. This equivalence may be related to the specific examples of alcohol that we provided (scotch and champagne). These flavours are not sweet, like candy or fruit. In addition, underage drinkers are most likely to drink malt beverages, beer or specific

Table 2 Correlates of interest in trying e-cigarettes if offered by a friend $(n=1125)$

\begin{tabular}{|c|c|c|}
\hline & \multicolumn{2}{|c|}{ Multivariable correlates } \\
\hline & $\begin{array}{l}\text { Number } \\
\text { interested/ } \\
\text { total number } \\
\text { in category (\%) }\end{array}$ & OR (95\% Cl) \\
\hline Overall & $83 / 1125$ (7.4\%) & \\
\hline \multicolumn{3}{|l|}{ Flavour } \\
\hline Tobacco/alcohol (Ref) & $14 / 451(3.1 \%)$ & 1.00 \\
\hline Menthol/candy/fruit & 69/674 (10.2\%) & $4.04(1.89 \text { to } 8.63)^{* * *}$ \\
\hline Perceived harm: mean (SD) & $2.18(0.65)$ & $0.48(0.31 \text { to } 0.75)^{* *}$ \\
\hline \multicolumn{3}{|l|}{ Sex } \\
\hline Male (Ref) & $41 / 561(7.3 \%)$ & 1.00 \\
\hline Female & $42 / 564(7.5 \%)$ & $1.68(0.90$ to 3.12$)$ \\
\hline Age: mean (SD) & $15.9(1.15)$ & $1.23(0.94$ to 1.59$)$ \\
\hline \multicolumn{3}{|l|}{ Race/ethnicity } \\
\hline Non-Hispanic white (Ref) & $65 / 859(7.6 \%)$ & 1.00 \\
\hline Non-Hispanic other race & $10 / 182(5.5 \%)$ & 0.99 (0.42 to 2.33$)$ \\
\hline Hispanic & $8 / 84(9.5 \%)$ & $1.14(0.36$ to 3.60$)$ \\
\hline \multicolumn{3}{|l|}{ Mother's education } \\
\hline High school or less (Ref) & 20/218 (9.2\%) & 1.00 \\
\hline $\begin{array}{l}\text { Some college or Associate's } \\
\text { degree }\end{array}$ & $17 / 186(9.1 \%)$ & $1.16(0.44$ to 3.05$)$ \\
\hline Bachelor's degree or more & $37 / 545(6.8 \%)$ & $0.82(0.35$ to 1.95$)$ \\
\hline Don't know & 9/176 (5.1\%) & 1.35 (0.45 to 4.08$)$ \\
\hline \multicolumn{3}{|l|}{ Region } \\
\hline Midwest (Ref) & $14 / 285(4.9 \%)$ & 1.00 \\
\hline Northeast & $13 / 154(8.4 \%)$ & $3.33(1.20 \text { to } 9.22)^{*}$ \\
\hline South & $42 / 545(7.7 \%)$ & 1.23 (0.52 to 2.92 ) \\
\hline West & $14 / 141(9.9 \%)$ & $2.17(0.74$ to 6.34$)$ \\
\hline \multicolumn{3}{|l|}{ Cigarette smoking } \\
\hline Never-smoker (Ref) & 13/1004 (3.3\%) & 1.00 \\
\hline Ever-smokert & $24 / 80(30.0 \%)$ & $3.16(1.45 \text { to } 6.89)^{* *}$ \\
\hline Current smokerł & 26/41 (63.4\%) & $7.82(2.86 \text { to } 21.32)^{* * *}$ \\
\hline \multicolumn{3}{|l|}{ E-cigarette use } \\
\hline Never-user (Ref) & 16/958 (1.7\%) & 1.00 \\
\hline Ever-usert & $34 / 109(31.2 \%)$ & $12.47(5.89 \text { to } 26.41)^{* * *}$ \\
\hline Current user $\ddagger$ & $33 / 58(56.9 \%)$ & $25.75(10.30 \text { to } 64.36)^{* * *}$ \\
\hline \multicolumn{3}{|c|}{$\begin{array}{l}{ }^{*} p<0.05,{ }^{* *} p<0.01,{ }^{* * *} p<0.001 . \\
\dagger \text { At least one time but not in past } 30 \text { days. } \\
\text { ¥At least one time in past } 30 \text { days. } \\
\text { Ref, reference category. }\end{array}$} \\
\hline
\end{tabular}


brands of liquors. ${ }^{16}$ Interest may have been higher if we had described alcoholic flavours that correspond to these products or brands (such as 'Jack Daniel's whiskey'). In a previous study with adolescent males conducted in 2011, we found no difference between interest in flavoured e-cigarettes, described as 'chocolate, mint, apple, etc', and non-flavoured e-cigarettes. ${ }^{15}$ However, we suspect this result was partly due to an unclear comparison group (which we described in that study as simply 'an e-cigarette' with no flavour specified) and lower levels of awareness of and interest in e-cigarettes overall in 2011 than in 2014-2015. . $^{15}$

Adolescents perceived that e-cigarettes with fruit flavours were less harmful than those with tobacco flavour. After controlling for other factors, they held similar beliefs about menthol and candy. Mediation analyses showed that perceived harmfulness explained some of the association between flavour and interest. Risk beliefs are a central predictor of many adult and adolescent health behaviours, including smoking. ${ }^{17-19}$ An important developmental difference is that, although adults treat some high-risk behaviours as categorically off-limits, adolescents weigh the pros and cons of even very dangerous activities. ${ }^{20}$ Thus, the presence of flavours may not only mask harsh taste but also reduce perceptions of harm, contributing to interest in e-cigarette experimentation. Perceived harmfulness may also potentially serve as a point of intervention to educate adolescents about the harms of all nicotine-containing products. The FDA's planned implementation of warning labels on nicotinecontaining e-cigarettes and accessories may draw adolescents' attention to possible harms from use.

Strengths of our study include that we used a betweenparticipants experimental design with a national probability sample of adolescents from the USA. The experimental design allows for causal inference and enables us to conduct mediation analysis without concerns about temporality. Limitations include that the description of e-cigarettes that our study used may not match current terminology, particularly given rapid development of new products in the marketplace. The phone survey mode prevented us from showing participants images of different models and brands of vaping devices to aid comprehension. While some respondents may not have fully understood the term menthol, interviewers offered an explanation if asked. Finally, our response rate was $66 \%$ and our sample had few smokers or e-cigarette users, although this is understandable given our focus on adolescents who are susceptible to initiation.

E-cigarettes remain in public health limbo as scientists try to understand their harm reduction potential. While we wait for this research to mature, adolescents are experimenting with ecigarettes in increasing numbers, ${ }^{7}$ and some physicians are even recommending e-cigarettes to adolescents as a way to quit smoking. ${ }^{21}$ This study contributes to the literature by describing adolescents' responses to particular flavours and examining the relationship between response and perceived harm. However, additional research is needed to understand factors that generate adolescents' interest beyond flavour descriptors. These factors may include enticing e-liquid names (eg, Fairy Nectar), novelty of flavours and packaging, and perceptions of luxury and prestige brands. The availability of and attraction to flavoured ecigarettes may contribute to product interest among adult cigarette smokers who can use e-cigarettes to quit smoking or engage in complete product substitution; thus, an outright ban on flavours could have adverse effects on overall harm reduction efforts. However, flavoured e-cigarettes may also be contributing to surging rates of adolescent experimentation. This trend is troubling given that the nicotine in e-cigarettes can lead to

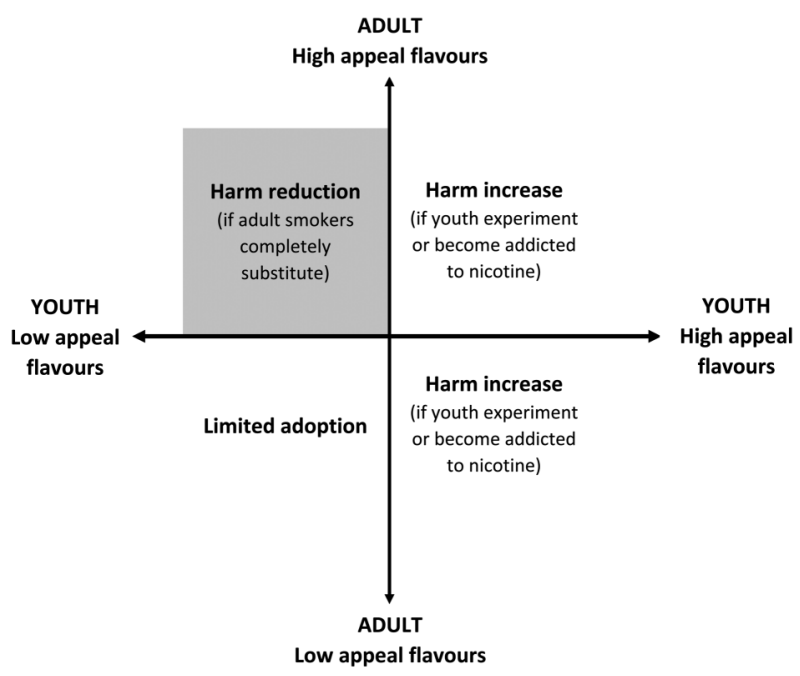

Figure 3 Ideal flavours for harm reduction among adult smokers and prevention of use among youth.

addiction or cause problems in adolescent brain development. ${ }^{22}$ An intriguing possibility is that some flavours may appeal to adults but have only minimal youth appeal, allowing for ecigarettes to serve a vehicle for harm reduction (shaded quadrant in figure 3). For example, it may be possible to pair tobacco flavour with other non-sweet flavours that have minimal youth appeal, for example, a spicy or piquant tobacco flavour. In the meantime, the public health community should work to restrict e-cigarette advertisements in media that reach large numbers of young people; this may be particularly important when those advertisements describe e-cigarettes with the sweet and minty flavours we found to be most enticing to adolescents or when e-liquid names (eg, Lemonade Delight) or images on product packaging (eg, icicles) conjure these flavours. Despite the fact that nationwide restrictions on flavours in e-cigarettes were struck from the final version of FDA's regulation, there is still room for public health efforts. Such efforts should focus on restricting the accessibility of flavoured tobacco products to youth locally (eg, Chicago's ban on the sale of flavoured tobacco products within 500 feet of schools) $)^{23}$ and strongly enforcing new regulations (eg, FDA's deeming rules and California's 'Tobacco 21' initiative) that ban the sale of ecigarettes to individuals under 18 and 21 , respectively. ${ }^{6}{ }^{24}$

\section{What this paper adds}

- Prior research suggests substantial interest in flavoured cigarettes and other flavoured tobacco products among adolescents.

- Rising interest in e-cigarettes among youth may be partially related to the thousands of available flavours, despite the potential harmful effects of flavourants.

- Few national studies have examined adolescents' preferences among specific flavours or whether the mediators of the relationship between flavours and interest in use vary by specific flavour.

- This study demonstrates that adolescents are not equally interested in all non-tobacco flavours and that perceived harm is one driver of the relationship between some flavours and interest in use. 
Contributors JKP conceptualised the experiment, developed the survey items and led the writing. NTB and KMR conceptualised the experiment, substantially contributed to survey item development, oversaw the project and provided substantial feedback on writing.

Funding Research reported in this publication was supported by P50CA180907 from the National Cancer Institute and the FDA Center for Tobacco Products (CTP).

Disclaimer The content is solely the responsibility of the authors and does not necessarily represent the official views of the National Institutes of Health (NIH) or the Food and Drug Administration.

Competing interests None declared.

Ethics approval University of North Carolina Institutional Review Board (IRB).

Provenance and peer review Not commissioned; externally peer reviewed.

\section{REFERENCES}

1 Carpenter CM, Wayne GF, Pauly JL, et al. New cigarette brands with flavors that appeal to youth: tobacco marketing strategies. Health Aff 2005;24:1601-10.

2 King BA, Tynan MA, Dube SR, et al. Flavored-little-cigar and flavored-cigarette use among U.S. middle and high school students. J Adolesc Health 2014;54:40-6.

3 Feirman SP, Lock D, Cohen JE, et al. Flavored tobacco products in the United States: a systematic review assessing use and attitudes. Nicotine Tob Res 2016;18:739-49.

4 Ambrose BK, Day HR, Rostron B, et al. Flavored tobacco product use among US youth aged 12-17 years, 2013-2014. JAMA 2015;314:1871-3.

5 Family Smoking Prevention and Tobacco Control Act. United States, 2009. Pub.L. 111-31, H.R. 1256. http://www.fda.gov/TobaccoProducts/ GuidanceComplianceRegulatoryInformation/ucm262084.htm (accessed Jun 2016).

6 Deeming Tobacco Products To Be Subject to the Federal Food, Drug, and Cosmetic Act, as Amended by the Family Smoking Prevention and Tobacco Control Act; Restrictions on the Sale and Distribution of Tobacco Products and Required Warning Statements for Tobacco Products. United States, 2016. Document number 201610685. https://federalregister.gov/a/2016-10685 (accessed May 2016).

7 Singh T, Arrazola RA, Corey CG, et al. Tobacco use among middle and high school students-United States, 2011-2015. MMWR Morb Mortal Wkly Rep 2016;65:361-7.

8 Zhu SH, Sun JY, Bonnevie E, et al. Four hundred and sixty brands of e-cigarettes and counting: implications for product regulation. Tob Control 2014;23(Suppl 3):iii3-9.

9 Hyland A, Conway K, Borek N, et al. Highlighted findings from wave 1 of the Population Assessment of Tobacco and Health (PATH) study. Society for Research on Nicotine and Tobacco Annual Meeting; 3 March 2016; Chicago, IL.
10 Corey CG, Ambrose BK, Apelberg BJ, et al. Flavored tobacco product use among middle and high school students-United States, 2014. MMWR Morb Mortal Wkly Rep 2015;64:1066-70.

11 Kong G, Morean ME, Cavallo DA, et al. Reasons for electronic cigarette experimentation and discontinuation among adolescents and young adults. Nicotine Tob Res 2015;17:847-54.

12 Allen JG, Flanigan SS, LeBlanc $M$, et al. Flavoring chemicals in e-cigarettes: diacetyl, 2,3-pentanedione, and acetoin in a sample of 51 products, including fruit-, candy-, and cocktail-flavored e-cigarettes. Environ Health Perspect 2016:124:733-9.

13 Lerner CA, Sundar IK, Yao H, et al. Vapors produced by electronic cigarettes and e-juices with flavorings induce toxicity, oxidative stress, and inflammatory response in lung epithelial cells and in mouse lung. PLOS ONE 2015;10:e0116732.

14 Shiffman S, Sembower MA, Pillitteri JL, et al. The impact of flavor descriptors on nonsmoking teens' and adult smokers' interest in electronic cigarettes. Nicotine Tob Res 2015;17:1255-62.

15 Pepper JK, Reiter PL, McRee AL, et al. Adolescent males' awareness of and willingness to try electronic cigarettes. J Adolesc Health 2013;52:144-50.

16 Siegel $\mathrm{M}$, Chen $\mathrm{K}$, DeJong $\mathrm{W}$, et al. Differences in alcohol brand consumption between underage youth and adults-United States, 2012. Subst Abus 2015;36:106-12.

17 Brewer NT, Chapman GB, Gibbons FX, et al. Meta-analysis of the relationship between risk perception and health behavior: the example of vaccination. Health Psychol 2007;26:136-45.

18 Brewer NT, Weinstein ND, Cuite CL, et al. Risk perceptions and their relation to risk behavior. Ann Behav Med 2004;27:125-30.

19 Song AV, Morrell HE, Cornell JL, et al. Perceptions of smoking-related risks and benefits as predictors of adolescent smoking initiation. Am J Public Health 2009;99:487-92.

20 Reyna VF, Farley F. Risk and rationality in adolescent decision making: implications for theory, practice, and public policy. Psychol Sci Public Interest 2006:7:1-44

21 Pepper JK, Gilkey MB, Brewer NT. Physicians' counseling of adolescents regarding e-cigarette use. J Adolesc Health 2015;57:580-6.

22 Dwyer JB, McQuown SC, Leslie FM. The dynamic effects of nicotine on the developing brain. Pharmacol Ther 2009;122:125-39.

23 Tobacco Control Legal Consortium. Chicago's regulation of menthol flavored tobacco products: a case study. 2015. http://publichealthlawcenter.org/sites/default/ files/resources/tclc-casestudy-chicago-menthol-2015.pdf (accessed Mar 2016).

24 Tobacco products: minimum legal age. State of California, 2016. Senate bill number SBx2-7. https://leginfo.legislature.ca.gov/faces/billTextClient.xhtml?bill_ $\mathrm{id}=201520162$ SB7 (accessed June 2016). 